\title{
Scaling Analysis of Magnetic Field Tuned Phase Transitions in One-Dimensional Josephson Junction Arrays
}

\author{
Watson $\mathrm{Kuo}^{1,2}$ and C. D. Chen ${ }^{2}$ \\ ${ }^{1}$ Department of Physics, National Tsing-Hua University, Hsin-Chu, 300, Taiwan, ROC \\ ${ }^{2}$ Institute of Physics, Academia Sinica, Nankang, 115, Taipei, Taiwan, ROC
}

(March 12, 2001)

\begin{abstract}
We have studied experimentally the magnetic field-induced superconductor-insulator quantum phase transition in onedimensional arrays of small Josephson junctions. The zero bias resistance was found to display a drastic change upon application of a small magnetic field; this result was analyzed in context of the superfluid-insulator transition in one dimension. A scaling analysis suggests a power law dependence of the correlation length instead of an exponential one. The dynamical exponents $z$ were determined to be close to 1 , and the correlation length critical exponents were also found to be about 0.3 and 0.6 in the two groups of measured samples.

PACS numbers: 73.40.Gk, 73.23.Hk, 74.50.+r
\end{abstract}

A 1D array of small Josephson junctions (JJ) provides an ideal testing ground for the $T=0$ quantum phase transition [1,2]. Theoretically, a $d$-dimensional JJ array can be mapped to the classical $(d+1)$-dimensional $X Y$ model, however, the types of governing phase transitions may not be the same in different dimensions [3,4]. In the 2D $X Y$ model, for instance, the exponentially dependent correlation length of KosterlitzThouless-Berezinskii(KTB) transition should lead to scaling properties different from those in a 3D model [5]. While scaling properties in 2D JJ arrays have been shown to exhibit an correlation length which corresponds to an underlying power law [6.7], no 1D arrays experiment has been reported to date. In this study we investigate the scaling behavior in 1D JJ arrays, and report a power law dependent correlation length. In addition, correlation length exponents $\nu$ are determined at approximately 0.3 and 0.6 .

As shown in Fig. 1 (a), the measured arrays were composed of I-shaped aluminum islands, whose sizes as defined by electron beam lithography were on the order of $1 \mu \mathrm{m}$. Each Al island has two tunnel junctions connected in parallel to its nearest neighbors, and forms a SQUID which is referred to as a unit cell. Being fabricated on the same chip, each group of measured arrays (denoted as group A and B) having different cell numbers, $N$, should have almost similar controlled parameters. Thus, length dependence of the phase transition can be investigated. The normal state resistances $R_{T}$ of each cell for the arrays, as listed in Table 1, are determined at high bias, $V>N\left(2 \Delta^{0} / e\right)$. The resistances for arrays in a group are quite similar, confirming the uniformity of the fabri- cated arrays.

When magnetic field $B$ is applied perpendicularly to SQUID loops with area $A$, the Josephson coupling energy $E_{J}$ can be tuned periodically as $E_{J}=E_{J}^{0} \cos \left(\pi A B / \Phi_{0}\right)$. The zero field Josephson coupling $E_{J}^{0}$ is determined using the $T=0$ Ambegaokar-Baratoff formula $E_{J}^{0}=$ $\left(\Delta^{0} / 2\right)\left(R_{Q} / R_{T}\right)$ with a superconducting gap $\Delta^{0}$ of about $200 \mu \mathrm{eV}$, and a resistance quantum $R_{Q}$ of $h / 4 e^{2} \approx 6.5 \mathrm{k} \Omega$. Accordingly, the $E_{J}^{0}$ values are approximately $130 \mu \mathrm{eV}$ for arrays in group $\mathrm{A}$, and $650 \mu \mathrm{eV}$ for arrays in group B. From the SEM image one can estimate the junction area, and infer a junction capacitance $C$ of $3.0 \pm 0.8 \mathrm{fF}$ by using a specific capacitance of $45 \mathrm{fF} / \mu \mathrm{m}^{2}$ [8]. With this capacitance, the charging energy for Cooper pairs, $E_{C P} \equiv(2 e)^{2} / 2 C$, is about $106 \pm 35 \mu \mathrm{eV}$, giving $E_{J}^{0} / E_{C P}$ values of about 6 and 1.3 for arrays in groups $\mathrm{A}$ and $\mathrm{B}$, respectively.

The transport measurements were conducted in a dilution refrigerator at temperatures ranging between $40 \mathrm{mK}$ and $1.5 \mathrm{~K}$. Samples were mounted inside an electromagnetic-field-sealed compartment within an oxygen-free copper holder. Any external noise was carefully prevented from reaching the sample compartment. The signal leads were filtered by $\pi$-section lowpass feedthrough filters, which were on the top of the cryostat and also with ThermoCoax cables from room temperature to $\mathrm{mK}$ temperature of sample compartment. To minimize the line frequency noise, a battery-powered preamplifier mounted on the top of cryostat was used. Furthermore, taking advantage of the common mode noise rejection, the entire measurement circuit was placed symmetrically with respect to its ground. Zero bias resistance $R_{0}$ was taken from the slope of $I(V)$ characteristics at a very small bias, and it was further confirmed by using a lock-in technique at a frequency of $1.7 \mathrm{~Hz}$ with an excitation of about $20 \mathrm{nV}$. Sweeping a wide range of magnetic fields at $40 \mathrm{mK}$, we determined the periodicity of magnetoresistance oscillation to be 9.15 Gauss. With this period $\Delta B$, we denote the field as a dimensionless filling number $f=B / \Delta B$, which represents an average number of flux quantum in one cell. At integer values of $f$, the arrays are most conductive with $R_{0}$ at a minimum, while at half integer $f$-values, the arrays become most resistive with $R_{0}$ at a maximum.

Figure 11(b) shows $I(V)$ characteristics for array A1 measured at $f=0 \sim 0.5$. For array A1 at $f=0$ (i.e. the most superconducting curve), deviations of the su- 
percurrent of consisting junctions are quite small, reaffirming the uniformity of these arrays. However, even at $f=0$, the array is not truly superconducting, but has a finite zero bias resistance of about $0.9 \mathrm{k} \Omega$. The supercurrent decreases with $f$ and diminishes at $f \simeq 0.45$, above which the supercurrent-type structure turns into a Coulomb-blockade-type structure, with zero bias resistance reaching a maximum value of about $17 \mathrm{M} \Omega$ at $f=0.5$. The evolution from one structure to the other is best represented by Fig. 11(c), which shows a smooth crossover from dip to hump structure with differential resistance $R_{d} \equiv d V / d I$ as a function of bias voltage, and with a flat $R_{d}(V)$ curve separating the two limits.

Due to a smaller $E_{J}^{0} / E_{C P}$ value, the $I(V)$ characteristics for array B1, depicted in Fig. 2(a), have a higher $R_{0}$ of about $100 \mathrm{k} \Omega$ at $f=0$ and $50 \mathrm{G} \Omega$ at $f=0.5$. The evolution of $I(V)$ characteristics, from the supercurrenttype structure to Coulomb-blockade-type structure, for this array is quite different than that of array A1: As $f$ increases beyond $f=0.20$, a small Coulomb gap in the $I(V)$ characteristic appears in the begining, signifying competition between Josephson coupling and Coulomb blockade of Cooper pair tunneling. This behavior can be clearly seen in Fig. 2(b), which shows, in addition to the simple dip and hump structures (as in Fig. 11(c)), a coexistence of the dip and hump structures in the $R_{d}(V)$ curves. From these $R_{d}(V)$ curves, supercurrent and Coulomb blockade thresholds are plotted as a function of the filling numbers and are shown in Fig. 2(c). Note that the two curves cross each other, a feature different from that reported in Ref. [1] with a considerably larger $E_{J}^{0} / E_{C P}$ value (about 6.1) than those of our arrays in group B.

The temperature dependences of zero bias resistance at various filling numbers for the arrays A2 and B1 are depicted in Fig. 3. For the most insulating case, the conductance fits standard Arrhenius form between $1 \mathrm{~K}$ and $150 \mathrm{mK}$ with an energy barrier of about $120 \mu \mathrm{eV}$, which is very close to $E_{C P}$. This suggests that the dynamics is dominated by simple thermal activation of Cooper pairs, because the strength of the Josephson coupling is suppressed to the minimum. At even lower temperatures, shorter arrays show a saturation of resistance probably due to the finite size effect. For the most superconducting case (i.e. $f=0$ ), the resistance decreases with decreasing temperature and levels off at $T<0.5 \mathrm{~K}$. At low temperatures with $f \neq 0, R_{0}(T)$ increases even with decreasing temperature. A close inspection reveals a separation point $R_{0}(T \rightarrow 0) \simeq R_{Q}$, above which $R_{0}(T)$ curves go upward with decreasing temperature (leveling off for the case of shorter arrays) and below which $R_{0}(T)$ curves simply level off. Similar results are reported by Haviland et al. [1]. We emphasize that this leveling off behavior is not due to any unwanted high-frequency noises in the measurement system for these reasons: 1 . the levelingoff temperatures for the $\mathrm{S}$ and I sides are not the same, and 2. the leveling-off temperatures for the arrays in the two groups are not the same. This leveling-off behavior cannot be accounted for by heating effects because the measured resistance is almost the same for both $\mathrm{AC}$ and DC measurements. There are possible reasons that may bring in a finite leveling-off zero bias resistance: the finite size effect, the vortex macroscopic quantum tunneling (MQT) [9], and the charge Coulomb interaction [10]. The finite size effect can be ruled out because the leveling-off temperature does not change in arrays with different lengths. Even if it does involve, it should set in at the same temperature for both $\mathrm{S}$ and I sides, contrary to the $R_{0}(T)$ curves. The vortex MQT, observed in wire experiments [9], has a pronounced effect in $1 \mathrm{D}$ systems and can result in broadening of superconductor transition. However, MQT should be exponentially suppressed by dissipation, which is quantified as a ratio between the quantum resistance and the junction tunneling resistance, $R_{Q} / R_{T}$. Arrays in group $\mathrm{A}$ have a dissipation about five times larger than that of arrays in group B, and should have a diminishing vortex MQT. The fact that a higher leveling off temperature for arrays in group A suggests that MQT is not responsible for this behavior at this temperature.

The last effect seems to be a reasonable one if the charge soliton picture is taken into account. While the origin model [4] describing phase transitions in 1D Josephson junction arrays considered only an "on-site" Coulomb interaction, in reality, the long range interaction due to a nonzero island-to-ground capacitance $C_{0}$ cannot be ignored. For finite $C / C_{0}$, it has been shown 111 that the charge will spread out and extend to a characteristic charge soliton length of $\Lambda=\sqrt{C / C_{0}}$. The result of the long range Coulomb interaction may lead to finite array resistances at low temperatures, driving even the superconducting phase to a metallic one [10]. To investigate the effect of this charge soliton picture, we made nearby grounded electrodes along each array to control $C_{0}$. The distance between electrodes and arrays for samples in group $\mathrm{A}$ is 8 times longer than that for arrays in group $\mathrm{B}$, leading to a smaller $C_{0}$ and thus a longer soliton length. This is qualitatively consistent with our experimental finding that arrays in group A have higher leveling off temperatures than that of group B.

For $T>0.5 \mathrm{~K}$, the sign of $d R_{0} / d T$ changes from positive to negative value depending upon increasing filling numbers, signifying that the system undergoes a quantum superconductor-insulator transition. According to the theory of superfluid-insulator transition in 1D systems [13], right at critical point $f=f^{*}$, the resistance is linearly proportional to the temperature. Experimentally, $f^{*}$ is identified as the filling number where the extrapolation of $R_{0}(T)$ curve passes $R_{0}=0, T=0$ point. As a supplementary clue, we notice that at the base temperature, this critical filling number corresponds to an onset of Coulomb blockade threshold voltage (see Fig. 2(c)). We thus interpret these phenomena as evidence of 
a magnetic field-tuned SI phase transition with a critical filling number $f^{*}$ in our measured arrays.

In a non-interacting model, the Hamiltonian of a $1 \mathrm{D}$ array of small JJ can be mapped to a classical 2D $X Y$ model. Theoretically, the dimensionless coupling constant $K$, playing the role of the temperature in the classical model, is related to the charging energy $E_{C P}$ and Josephson coupling energy $E_{J}$ as, $K=\sqrt{E_{J} / 2 E_{C P}}$ in the quantum system [4]. The 2D $X Y$ model has a KTB type transition. Below the transition temperature $T_{\mathrm{KTB}}$, the spins form vortex-antivortex pairs, while above $T_{\mathrm{KTB}}$, the pairs dissociate and the whole system becomes a vortex plasma [5]. Note that the topological spin vortex in the 2D $X Y$ model represents the phase slip in 1D JJ arrays. In our system, the corresponding KTB transition takes place at critical coupling energy $E_{J}^{*}$, which is achieved by the tuning of external magnetic field. In the region $E_{J}<E_{J}^{*}$, corresponding to the pair-dissociation phase at $T>T_{\mathrm{KTB}}$, the long range order in phase vanishes, and phase fluctuations render insulating 1D JJ arrays. According to the model, the transition takes place at $E_{J}^{*} / E_{C P}=8 / \pi^{2} \simeq 0.81$ 3; however the experimental values are slightly larger than the theoretical one. Despite the scattering of $E_{J}^{*} / E_{C P}$ values in group $\mathrm{A}$, the values in group $\mathrm{B}$ are well consistent to one other. The difference between the two groups can be explained with the effect of dissipation. At different strength of dissipation, it is expected that the critical point of SI phase transition will also be different [12].

The finite-temperature scaling law of quantum phase transitions asserts that $\mathcal{O}\left(k, \omega, K, L_{T}\right)=$ $L^{d_{\mathcal{O}} / z} \mathcal{O}\left(k L_{T}^{1 / z}, \omega L_{T}, L_{T} / \xi_{T}\right)$, where $L_{T}=\hbar \beta$ is a finite length on the imaginary time axis. Some of the terms can be neglected: the wave vector $k$ is assumed zero, the scaling dimension $d_{\mathcal{O}}=2-d=1$ [13], and $\omega=0$ in DC measurements. We thus obtain a concise finite-size scaling form for zero bias resistance, $R_{0}(f, T)=T^{1 / z} \widetilde{R_{0}}\left(1 / T \xi^{z}\right)$, where $\xi$ is a function of $f$. To examine the correlation length dependence, we rewrite the scaling form as $R_{0}(\delta, T)=T^{1 / z} \widetilde{R_{0}}\left(T_{1} / T\right)$ where $\delta=\left(K-K^{*}\right) / K^{*} \sim$ $\left(f-f^{*}\right)$ is the distance from the transition point and $T_{1}$ are field dependent scaling parameters (see [15] for details). In this way, one obtains a clear power law dependence of $T_{1}$ on $\delta$ over one order of magnitude, suggesting a power law dependence of the correlation length. This is in contradiction to the exponential dependence predicted in KTB transition and other theories [14].

Based on this analysis, the correlation length $\xi$ can be written as $\xi=|\delta|^{-\nu}$ where $\nu$ is a critical exponent. Finally, the scaling law can be written with a scaling function $\widetilde{R_{0}}$ as

$$
R_{0}(f, T)=T^{1 / z} \times \widetilde{R_{0}}\left(\frac{\delta}{T^{1 / \nu z}}\right) .
$$

The exponent $\nu z$ is determined from the slope of the power law fitting. For the seven arrays measured, we find that the $\nu z$ are different varying from 0.30 to 0.60 for different arrays. Nevertheless, the same values of $\nu z$ are obtained from both $\mathrm{S}$ and I sides. Assuming a $z$ value of unity and using Eq. (1) with $\nu z$ and $f^{*}$ obtained from above, the scaling curves shown in Fig. 4 can be obtained. In addition, the scaling function form is found to be $\widetilde{R_{0}}(x) \propto e^{\alpha x}$, with $\alpha \simeq \pm 10$ and \pm 2.5 (in unit of $\mathrm{K}^{1 / \nu z}$, '+' for I-phase and '-' for S-phase), for arrays in groups A and B, respectively. This form for resistance is similar to results deduced from variable-range hopping(VRH) for the Bose glass [14]. However, the VRH mechanism should not be accounted for our system since the exponent on $T$ is larger than 1 . The fact that the scaling function in one phase is symmetrical to that in the other phases suggests that the $\mathrm{S}$ phase and the I phase plays a dual role at zero bias. To refine the critical filling number and the scaling exponents, one begins with a trial scaling form, namely, $R_{0}(f, T)=A T^{1 / z} \exp \left(\kappa(f) / T^{1 / \nu z}\right)$. Вy noting that $A$ is $f$-independent constant and that $\kappa$ is zero at $f^{*}$, the $\nu z$ and $f^{*}$ values can be unambiguously determined. The only adjustable parameter, $z$, can then be determined from the scaling curves. This parameter can be determined to a reasonable accuracy; a smaller $z$ would give better scaling on the S-side, whereas a larger $z$ would result in better scaling on the I-side. This finetuning procedure slightly modifies $\nu z$ and $f^{*}$ values, and gives a $z$ value of about $0.85 \pm 0.05$ for array B1.

To summarize, we observed a magnetic field-tuned SI phase transition in the system of 1D small Josephson junction arrays, and have, for the first time, made scaling analysis on such a system. Near the critical point, the $R_{0}(T)$ scaling analysis indicates a power law dependent correlation length. The exponents $\nu z$ are found to be 0.3 to 0.6 with $z$ close to 1 , implying an isotropy in spatial and time dimensions. The value of correlation length exponent $\nu$ contradicts what expected under current theory of $1 \mathrm{D}$ Boson system, $\nu \geq 2 / d$ and $\nu=\infty$ 14. The regions of lower temperature, where probably undermined complicated physics to make scaling fail, is away from the scope of the non-interacting model. These results suggest that certain important physics has not been unearthed in this system.

Fruitful discussions with N. Trivedi, L. Balents, A. Schakel, P. Phillips, S. K. Yip, C. M. Ho, and T. K. Lee are gratefully acknowledged. We have utilized facility at the National Nano Device Laboratories. This research was partly funded by the Nation Science Council No. 89-2112-M-001-033.

$\dagger \quad$ e-mail address: chiidong@phys.sinica.edu.tw

[1] E. Chow, P. Delsing, and D. B. Haviland, Phys. Rev. 
Lett. 81, 204(1998); D. B. Haviland, K. Andersson, and P. Agren, cond-mat/0001143

[2] A. van Oudenaarden and J. E. Mooij, Phys. Rev. Lett. 76 , 4947(1996); A. van Oudenaarden, S. J. K. Várdy, and J. E. Mooij, Phys. Rev. Lett. 77, 4257(1996)

[3] R. M. Bradley and S. Doniach, Phys. Rev. B 30, 1138(1984);

[4] S. L. Sondhi, S. M. Girvin, J. P. Carini, and D. Shahar, Rev. Mod. Phys. 69, 315(1997)

[5] J. M. Kosterlitz and D. J. Thouless, J. Phys. C 6, 1181(1973); J. M. Kosterlitz, J. Phys. C 7, 1046(1974)

[6] H. S. J. van der Zant, F. C. Fritschy, W. J. Elion, L. J. Geerligs, and J. E. Mooij, Phys. Rev. Lett. 69, 2971(1992)

[7] C. D. Chen, P. Delsing, D. B. Haviland, Y. Harada, and T. Claeson, Phys. Rev. B 51, 15645(1995)

[8] P. Delsing, T. Claeson, K. K. Likharev, and L. S. Kuzmin, Phys. Rev. B 42, 7439(1990)

[9] N. Giordano, Phys. Rev. Lett. 61, 2137 (1988)

[10] S. R. Renn and J.-M. Duan, Phys. Rev. Lett. 76, 3400 (1996); B. J. Kim, G. S. Jeon, M.-S. Choi and M. Y. Choi, Phys. Rev. B 58, 14524 (1998)

[11] K. K. Likharev and K. A. Matsuoka, Appl. Phys. Lett. 67, 3037 (1995)

[12] S. E. Korshunov, Europhys. Lett. 9, 107(1989); W. Zwerger, Europhys. Lett. 9, 421(1989)

[13] M. P. A. Fisher, G. Grinstein, and S. M. Girvin, Phys. rev. Lett. 64, 587(1990); M.C. Cha, M. P. A. Fisher, S. M. Girvin, M. Wallin, and A. P. Young, Phys. Rev. B 44, 6883(1991)

[14] M. P. A. Fisher, P. B. Weichman, G. Grinstein, and D. S. Fisher, Phys. Rev. B 40, 546(1989)

[15] The $T_{1}(\delta)$ dependence was determined in the following way: We searched for an $f$-dependent variable $T_{1}$ which could overlie the $R_{0} / T\left(T / T_{1}\right)$ curves for various $f$ near $f^{*}$ into a single curve.

FIG. 1. (a)The SEM image of an 1D JJ array. The overlapping areas between the ' $\mathrm{I}$ ' shaped islands are the tunnel junctions. The scale bar at the bottom of the image is $1 \mu \mathrm{m}$. (b) Evolution of $I V$ characteristics for arrays A2, from superconducting behavior to insulating behavior, at selected filling numbers between 0.0 and 0.5. (c) Dynamic resistance $R_{d}$ as a function of bias voltage show a crossover from superconducting (bottom, $f=0.42$ ) to insulating behavior (top, $f=0.50$ )

FIG. 2. $I V$ characteristics (a) and differential resistance $R_{d}(V)$ (b) for array B1 for $f=0 \sim 0.5$ at $40 \mathrm{mK}$. Notice the coexistence of the hump and dip structures; this is not seen in arrays in group A (c.f. Fig. 1(c)). The curves in (b) are shifted for clarity. (c) The $f$-dependence of supercurrent (solid square) and threshold voltage (open diamond) for array B1. Both are converted to the energy scale, i.e. $\hbar I_{C} / 2 e$ and $\mathrm{eV}$. The supercurrent is magnified 100 times.
FIG. 3. The $R_{0}(T)$ as a function of the filling number $f$ for the array A2(a) and B1(b). At about $0.5 \mathrm{~K}$ above, the array displays an $f$-tuned SI transition, whereas at low temperatures the $R_{0}(T)$ curves in the S-side level off or rise.

FIG. 4. $R_{0}(f, T)$ Scaling curve for array B1 for $0.5 \mathrm{~K}<T<1 \mathrm{~K}$ and $0<f<0.5$. The inset shows the scaling curve for array A2.

TABLE I. Some important parameters of the measured arrays. The arrays that are fabricated on the same chip and thus have similar junction parameters, are categorized into one group. Note the closeness between the critical filling numbers $f^{*}$ and the correlation length exponents $\nu$ for arrays in a group.

\begin{tabular}{|c|r|r|r|r|r|r|r|}
\hline \hline Sample & $\mathrm{A} 1$ & $\mathrm{~A} 2$ & $\mathrm{~A} 3$ & $\mathrm{~B} 1$ & $\mathrm{~B} 2$ & $\mathrm{~B} 3$ & $\mathrm{~B} 4$ \\
\hline$N$ & 49 & 29 & 14 & 100 & 75 & 50 & 30 \\
\hline$R_{T}$ & 0.9 & 1.1 & 0.9 & 5.0 & 4.7 & 4.3 & 4.9 \\
\hline$f^{*}$ & 0.41 & 0.41 & 0.45 & 0.20 & 0.25 & 0.26 & 0.27 \\
\hline$\nu z$ & 0.3 & 0.3 & 0.3 & 0.6 & 0.6 & 0.5 & 0.5 \\
\hline$E_{J}^{*} / E_{C}$ & 2.0 & 1.6 & 1.1 & 1.05 & 0.97 & 1.02 & 0.88 \\
\hline \hline
\end{tabular}




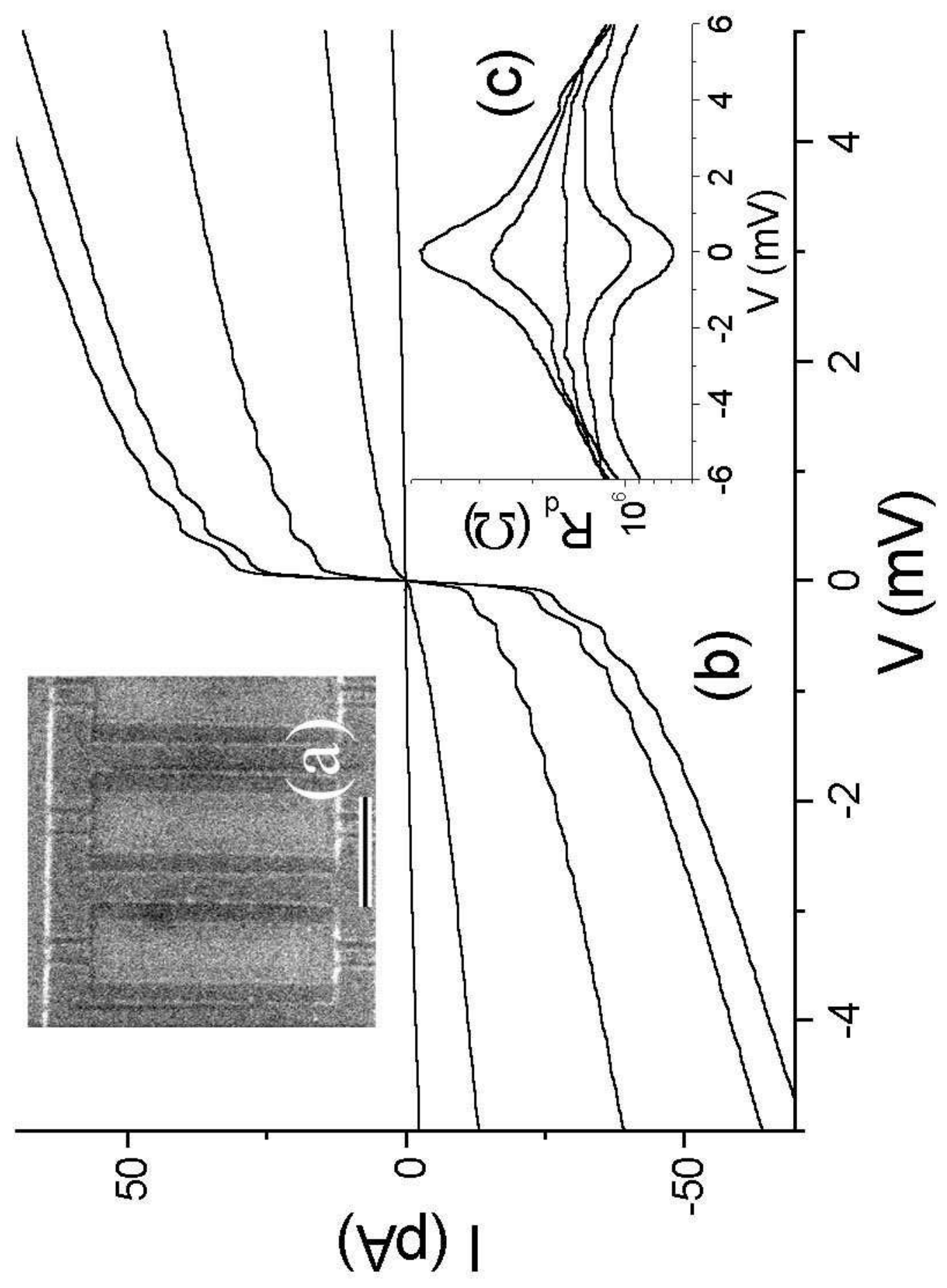




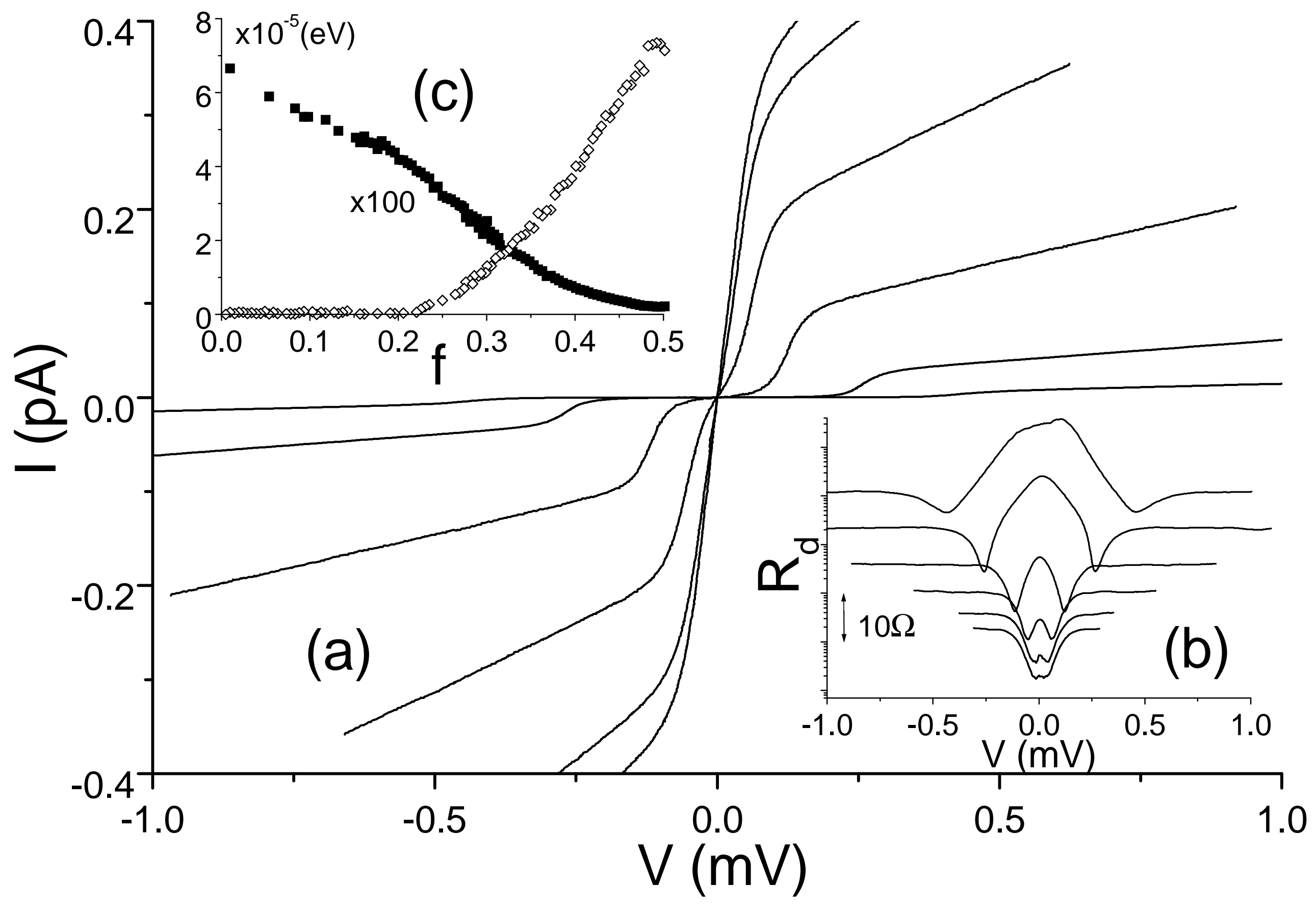




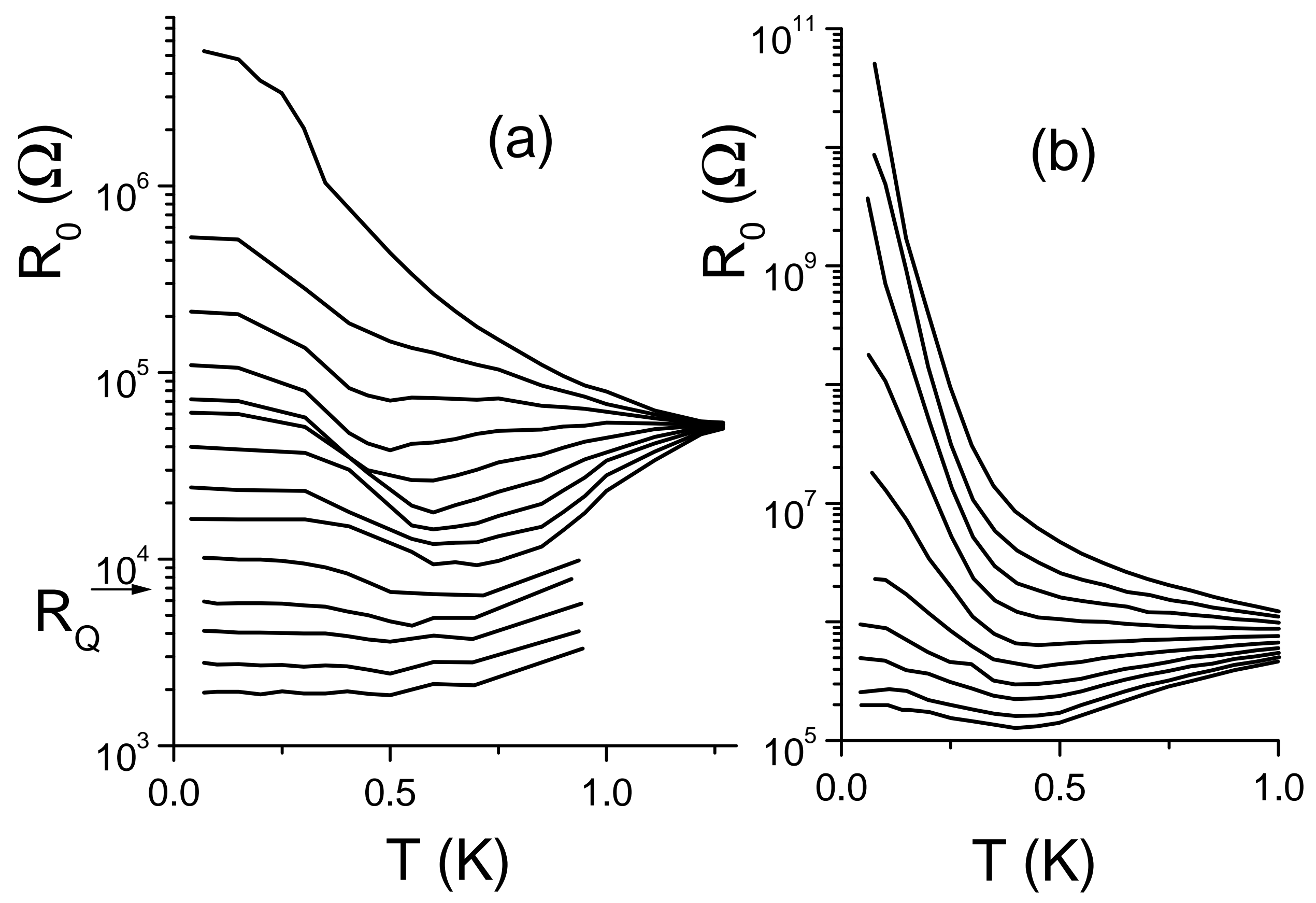




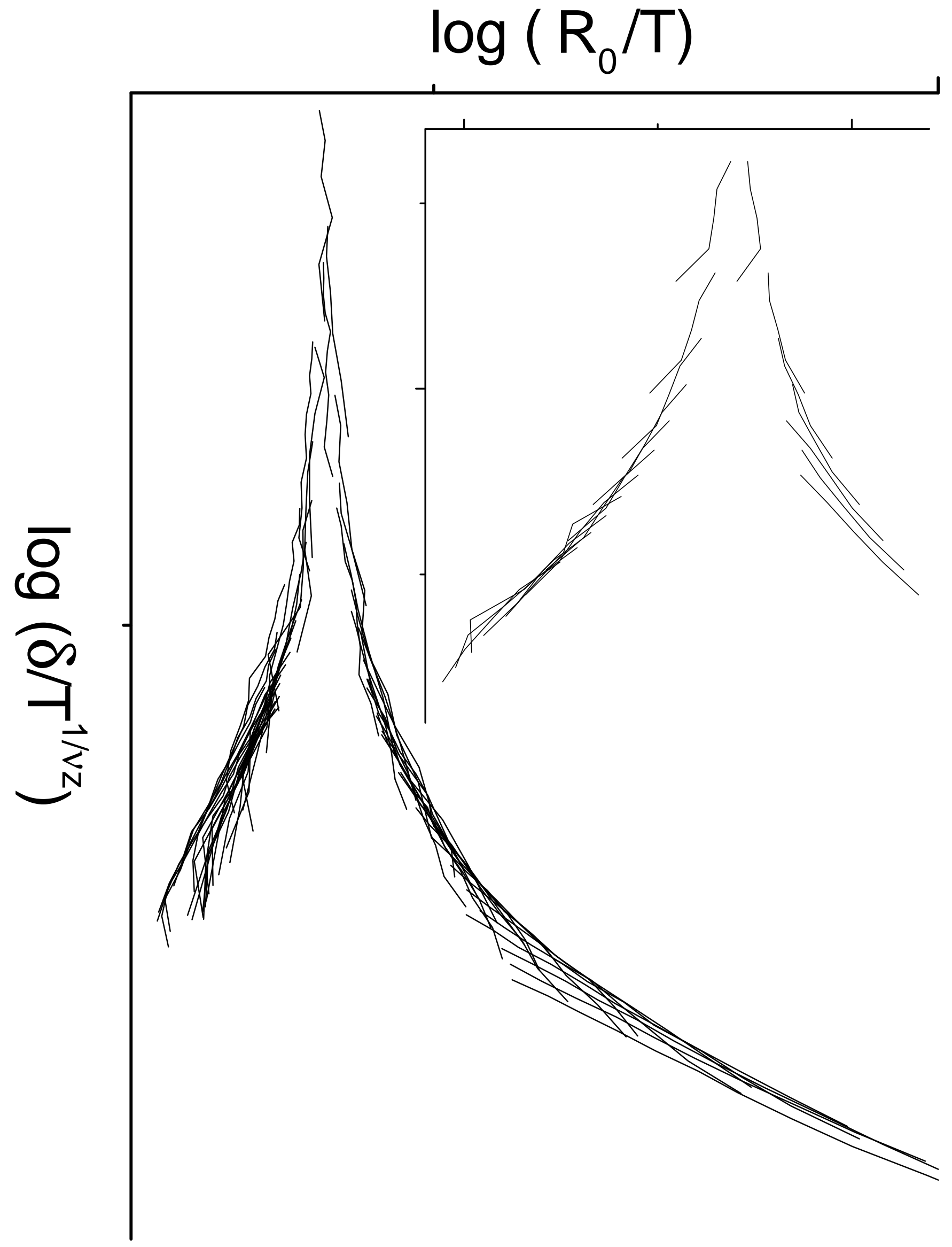

